\title{
EL TURISMO COMUNITARIO COMO HERRAMIENTA PARA EL DESARROLLO SOSTENIBLE DE DESTINOS SUBDESARROLLADOS
}

\author{
Francisco Orgaz Agüera ${ }^{1}$ \\ Universidad Tecnológica de Santiago (UTESA), República Dominicana \\ http://dx.doi.org/10.5209/rev_NOMA.2013.v38.42908
}

Resumen.-El turismo es uno de los principales sectores económicos a nivel internacional. Así, desde hace años, se viene trabajando en el desarrollo de nuevas formas de turismo, que se practican de forma sostenible, y tienen como finalidad mejorar el desarrollo socioeconómico de las comunidades locales a través de su potencial cultural, patrimonial y natural, así como mejorar la conservación de los recursos naturales, culturales y patrimoniales. En este sentido, el objetivo de esta investigación en analizar el turismo comunitario como herramienta para el desarrollo sostenible de destinos subdesarrollados. La metodología empleada ha consistido en una revisión de la literatura sobre turismo comunitario y desarrollo sostenible, y en la observación participante en destinos subdesarrollados con potencial turístico para el desarrollo del turismo comunitario.

Palabras clave- turismo comunitario; desarrollo sostenible; comunidades locales; cultura; recursos naturales.

\section{Community-based tourism as a tool for sustainable development in underdeveloped destinations}

Abstract.-Tourism is one of the most important economic sectors internationally. So, for some time, stakeholders have been working on the development of new forms of tourism. These new forms of tourism are practiced sustainably, and are aimed at improving the socio-economic development of local communities and to improve the conservation of natural, cultural and heritage resources. The objective of this research is to analyze community-based tourism as a tool for sustainable development of underdeveloped destinations. The methodology consisted of a review of the literature on community tourism and sustainable development, and in a fieldwork in underdeveloped destinations with tourism potential for the development of community-based tourism.

Keywords: community-based tourism; sustainable development; local communities; culture, natural resource

\section{Introducción.}

El turismo se configura como una de las principales industrias económicas a nivel internacional, siendo unos de los sectores más estudiados en ciencias sociales por numerosos autores e investigadores.

\footnotetext{
${ }^{1}$ Profesor en Turismo, Administración de Empresas y Medio Ambiente en la Universidad Tecnológica de Santiago (UTESA), República Dominicana. Doctorando en Turismo por la Universidad de Sevilla (España). Máster en Dirección y Planificación del Turismo por la Universidad de Sevilla. Diplomado en Turismo por la Universidad de Córdoba (España). Sus líneas de investigación son el turismo, medio ambiente y el desarrollo sostenible. Email: franorgaz@hotmail.com
} 
Así, desde hace años se viene trabajando en los destinos en el desarrollo de nuevas formas de turismo alternativas al turismo de masas. Son formas que tienen por finalidad, por un lado, respetar y conservar la naturaleza, y, por otro lado, mejorar el desarrollo socioeconómico de las comunidades locales. Es decir, se configuran como un turismo sostenible en el destino donde se desarrolla. Así, entre estas nuevas formas de turismo alternativas encontramos el turismo comunitario (Ruiz et al., 2008), el ecoturismo (Baralet al., 2008), el turismo gastronómico (Maket al., 2012), etc.

En estetrabajonos centramos en el turismo comunitario, turismo a través del cual la población local busca mejorar su situación socioeconómica y conservar los recursos naturales de su territorio, a través del contacto establecido entre la comunidad local y el turista (López-Guzmán y Sánchez Cañizares, 2009a). Además, el turista también interactúa con las organizaciones que prestan el servicio turístico, con el entorno donde se desarrolla la actividad turística y con el contexto en el que se desarrolla el propio servicio (Castellanos Verdugo y Orgaz Agüera, 2013), y por lo tanto, puede propiciar que aparezca algún tipo de impacto cultural entre la propia comunidad local y el visitante, o, también, medioambiental en el destino, sino se tienen en cuenta aspectos como la capacidad de carga (Nae-Wen y Pei-Hun, 2009).

En concreto, el objetivo de esta investigación es analizar el turismo comunitario como herramienta para el desarrollo sostenible de los destinos subdesarrollados o en vías de desarrollo.

La metodología empleada para elaborar este estudio parte de fuentes secundarias, en concreto, de una profunda revisión de la literatura científica que diversos autores han desarrollado en el campo del turismo comunitario y el desarrollo sostenible, así como la observación participante a través de diversas "vivencias" en países subdesarrollados con potencial para el desarrollo del turismo comunitario.

Para ello, este artículo se estructurará, tras esta introducción, en un segundo apartado donde se realiza una introducción al turismo comunitario; en un tercer apartado se desarrolla una introducción al desarrollo sostenible en el turismo; en un cuarto apartado se tratan las conclusiones de este estudio; y, finalmente, en un quinto apartado, se muestran las referencias bibliográficas consultadas para elaborar esta investigación.

\section{Introducción al turismo comunitario}

La investigación relacionada con el turismo comunitario ha sido objeto de estudio por numerosos académicos e investigadores, identificando Casas Jurado et al. (2012) proyectos sobre turismo comunitario en diversos países y/o destinos: Bolivia (Palomo Pérez, 1998), baja California (Bringas Rábago e Israel, 2004), Ecuador (Ruiz et al., 2008), El Salvador(López Guzmán y Sanchez Cañizares, 2009a), Nicaragua (López Guzmán y Sanchez Cañizares, 2009b) y Costa Rica (Trejos y Matarrita-Cascante, 2010).

En este mismo sentido, una revisión de la literatura más profunda, refleja que el turismo comunitario ha sido objeto de estudio para numerosos académicos e investigadores en muchos otros países: Chile (Cruz Blasco, 2012), Galápagos (Ruiz Ballesteros y Cantero Martín, 2011),Perú (Zorn y Farthing, 2007), Kenya 
(Manyara y Jones, 2007), México (Juárez Sanchez y Ramírez Valverde, 2008), Japón (Hiwasaki, 2006), Brasil (GuerreiroMarcon, 2007), Australia (Dyeret al., 2003), Panamá (CioceSampaioet al., 2007), Belize (Timothy y White, 1999), Botswana (Mbaiwa, 2005), Hawái (Wyllie, 1998), China (Ying y Zhou, 2007), Italia (Iorio y Wall, 2012), Turquía (Alaeddinoglu y Can, 2011), Tailandia (Ishii, 2012), Rumania (Iorio y Corsale, 2013), Uganda (Lepp, 2007), Malasia (Harunet al., 2012), Namibia (Lapeyre, 2010), Dominica (Patterson et al., 2004), Tanzania (Nelson et al., 2010), Canadá (Stewart y Draper, 2009), Cabo Verde (López Guzmán et al., 2011), Camboya (Reimer y Walter, 2013), India (Chakravarty y Irazabal, 2011), Suráfrica (Giampiccoli y Kalis, 2012), Fiji (Farrelly, 2011) o Madagascar (Sommervilleet al., 2010).

Por tanto, esto muestra la importancia que ha adquirido esta forma de turismo en los destinos, y su posterior estudio por parte de la comunidad científica. Esto, además, evidencia el proceso de potenciación de las comunidades locales, que según Casas Jurado et al., (2012), se están desarrollando, en los últimos años, en países subdesarrollados mediante el turismo comunitario. Es decir, la cultura, el patrimonio y los recursos naturales locales están siendo usados por las comunidades locales para mejorar el desarrollo socioeconómico del destino.

En cuanto al origen del turismo comunitario, es en la obra de Murphy (1985) cuando aparece por primera vez el término de turismo comunitario. A partir de entonces, muchos han sido los investigadores que han venido definiendo este concepto, denominado tambiéncon otros términos, como por ejemplo "community-based tourism" (Ruiz et al., 2008). En este sentido, para el Fondo Mundial Para la Naturaleza - World Wide FundforNature, en inglés - (WWF, 2001:4) el turismo comunitario hace referencia a"aquel tipo de turismo en el que la comunidad local tiene un control sustancial de, y participa en, su desarrollo y manejo, y una importante proporción de los beneficios se quedan en la comunidad". Por este motivo, Scheyvens (1999) sugiere que el término turismo comunitario se use únicamente para los casos donde los miembros de la población local tienen un alto control de las actividades y los beneficios, a diferencia de aquellos otros que son controlados principalmente por touroperadores externos o por el Gobierno (Trejos, 2009).

Siguiendo a Lopez-Gúzman y Sanchez Cañizares (2009a:89), el turismo comunitario es una actividad que "se fundamenta en la creación de productos turísticos bajo el principio básico de la necesaria participación de la comunidad local". En este sentido, para Casas Jurados et al., (2012:93), el turismo comunitario se refiere a aquel turismo que"está basado enla comunidad local" y "que pretende reducir el impacto negativo y reforzar los impactos positivos del turismo en la naturaleza".

Según Cruz Blazco (2012:131), esta forma de turismo se presenta como una "oportunidad de puente entre la rentabilidad económica, la conservación del medio ambiente y el respecto sociocultural". En este aspecto, para Ruiz et al., (2008) el turismo comunitario no se asocia a un producto turístico específico, pero si se vincula con la actividad ecoturística, que se define, siguiendo aJalani (2012), como aquel tipo de turismo que se desarrolla en áreas naturales y tiene como objetivo la conservación de las zonas naturales y el desarrollo de las comunidades locales. Aunque, según Ruiz et al., (2008), el turismo comunitario 
se desarrolla en otras formas de turismo, como por ejemplo en el turismo cultural, de aventura, etc.

En este sentido, según López-Guzmán y Sanchez Cañizares (2009a), el turismo comunitario está apadrinado por diversas instituciones internacionales, entre las que destacan la Organización Mundial del Turismo (OMT, 2002) y el Fondo Mundial Para la Naturaleza (WWF, 2001). Siguiendo a Ruiz et al., (2008), este turismo también es apoyado por otros stakeholders locales. Aunque, esta forma de turismo ha sido objeto de crítica también por algunos autores (Ruizet al., 2008) por razones neocolonialistas, por su excesiva centralización en lo medioambiental, por su dependencia de las ONGs (Manyaraet al., 2006), y por las deficiencias existentes desde la perspectiva del desarrollo de la comunidad local (Blackstock, 2005).

Así, según Hiwaski (2006:677), este turismo tiene varios objetivos, destacandoaquellos relacionados con la conservación de los recursos naturales, patrimoniales y culturales, el desarrollo socioeconómico de las comunidades locales, y la calidad recibida por la demanda turística.

Así, según López-Guzmán y Sanchez Cañizares (2009a), el turismo comunitario se compone por tres bloques: El primero lo formarían los stakeholders encargados de la planificación turística (empresas, guías, gobierno local, oficinas de turismo, etc.); el segundo bloque lo conformarían las empresas de servicios directas (hoteles, restaurantes, etc.); y, por último, el tercer grupo estaría formado por otras empresas que apoyan el turismo en el destino (medios de transporte, tiendas de suvenir, turismo activo, etc.).

Por otro lado, la puesta en marcha de este turismo también puede generar beneficios y costos. Así, según Manyara y Jones (2007), el turismo comunitario mejoraría el desarrollo socioeconómico de la población local, además de fomentar la conservación de la cultura y naturaleza local (López-Guzmán y Sanchez Cañizares, 2009a). Para Casas Jurado et al., (2012), esta forma de turismo se configura como una herramienta de lucha contra la pobreza, que tiene importantes efectos multiplicadores en el Producto Interior Bruto (PIB), y que permite conservar el patrimonio natural, histórico y la identidad étnica de un destino turístico. Por su parte, el desarrollo de este turismo puede presentar algunos impactos negativos, como por ejemplo la contaminación ambiental, el deterioro de la cultura, etc. Por esta razón, este turismo debe planificarse adecuadamente, bajo la coordinación conjunta de todos los stakeholders implicados, con la finalidad de obtener beneficios para el destino (sobre todo para la población local) y controlar todos aquellos costes o impactos negativos que esta actividad puede generar en el lugar.

\section{Introducción al desarrollo sostenible en el turismo}

El origen del desarrollo sostenible proviene de la Comisión Mundial para el Medio Ambiente y el Desarrollo (World CommissiononEnvironment and Development), cuando aprobó en abril de 1987, el informe Brundtland "Nuestro Futuro Común" (OurCommonFuture, en inglés) (Brundland, 1987). En este informe, elaborado por la comisión que encabezó la Doctora Gro Harlem Brundtland, se definió por primera vez el concepto de desarrollo sostenible como "el desarrollo que satisface las necesidades del presente sin 
comprometer la capacidad de las generaciones futuras de satisfacer sus propias necesidades". Posteriormente, empezaron a surgir las primeras medidas dirigidas a promover la sostenibilidad o el desarrollo sostenible en el turismo.

Desde entonces, el término desarrollo sostenible se ha vuelto muy común, utilizándose en diversas áreas (económica, comercio, finanzas, medio ambiente, sociología, etc.) como concepto relacionado con la conservación medioambiental y el desarrollo socioeconómico. En el campo de la industria turística, han sido muchos investigadores lo que han tratado este término (Hunter, 1997; Godfrey, 1998; Tosun, 2001; Shenet al., 2008; Jarviset al., 2010; Waligoet al., 2013).

Así, Sancho (1998) comenta que la sostenibilidad está ligada a tres hechos importantes como son la calidad, la continuidad y el equilibrio, buscando así mejorar la calidad de vida de la población local, mantener la calidad del medio ambiente, promover mayor calidad de experiencia para el turista, asegurar la obtención de beneficios por parte de los empresarios turísticos, y conseguir mayores niveles de rentabilidad económica para los residentes (Puertas Cañaveral, 2007). Es decir, se busca obtener beneficios para todos los stakeholders locales, a la vez que se intenta no generar impactos negativos en el medio ambiente.

En este sentido, esta definición ha sido adaptada al concepto de turismo. Así, Sancho (1998) desarrolló una definición de turismo sostenible, que a su vez, se incorporóa la Agenda 21 de la Industria del Turismo y los Viajes: "Aquellas necesidades de los turistas y de las regiones anfitrionas del presente, además que preserva y promueve las oportunidades para el futuro. Está enfocado a un modelo de gestión de todos los recursos de tal forma que se satisfagan todas las necesidades económicas, sociales y estéticas al tiempo que se respeta la integridad cultural, los procesos ecológicos esenciales, la diversidad biológica y los sistemas de apoyo a la vida".

En general, han sido muchas las instituciones e investigadores que han establecido los principios teóricos del turismo sostenible, aunque para todas ellas la aplicación de los principios de sostenibilidad a la actividad turística nos puede llevar a hablar de los siguientes puntos:

- $\quad$ El turismo sostenible hace referencia a una forma de turismo que busca la satisfacción de las necesidades actuales de los turistas, la industria turística y las comunidades locales, sin comprometer la capacidad de satisfacer las necesidades de las generaciones futuras.

- No existe sostenibilidad si no viene dada de una manera integral, teniendo en cuenta muy diferentes aspectos y agentes implicados; es decir, la sostenibilidad no se centra sólo en la protección del medio ambiente, sino también en una eficiencia económica y una equidad social, y en la viabilidad a largo plazo de todas ellas.

- $\quad$ La sostenibilidad es aplicable a la totalidad de elementos presentes en el espacioturístico.

- $\quad$ El turismo sostenible sólo existe como producto/resultado de un triple balance positivo en marco del que se ha venido denominado como triangulo o dimensiones de la sostenibilidad. 
En este sentido la sostenibilidad turística contempla una serie de características en cada una de esas dimensiones. Por su parte, la sostenibilidad económica asegura un crecimiento del turismo eficiente, con lo que el empleo y la renta tendrían unos niveles satisfactorios, junto a un control de los impactos negativos y positivos sobre los recursos y la actividad (Puertas Cañaveral, 2007). Así, la actividad económica debeasegurarse a largo plazo, dejando unos beneficios en el área homogéneos en sudistribución (Canoves et al., 2006). Algunos de los criterios para lograr una sostenibilidad económica de la actividad turística pueden ser:

- Luchar contra aquellas formas de la actividad de gran coste, en relación con sus limitados beneficios económicos.

- Procurar que los beneficios derivados del turismo se extiendan lo más posible entre la población y los agentes locales.

- $\quad$ Proteger a las empresas locales de la competencia desleal por parte de grandes compañías internacionales con escaso compromiso en el destino.

- $\quad$ Asegurarse de que el turista paga un precio justo por su experiencia (relación calidad-precio).

Por otro lado, la sostenibilidad ecológica o ambiental asegura que el desarrollo turístico y los procesos ecológicos esenciales de la diversidad biológica y de los recursos biológicos sean compatibles (Puertas Cañaveral, 2007). Es decir, se debe dar un uso óptimo a los recursos ambientales, que son un elemento fundamental del desarrollo turístico, manteniendo los procesos ecológicos esenciales y ayudando a conservar los recursos naturales y la diversidad biológica (Canoves et al., 2006). Por consiguiente, hay que tener presente los servicios que el medio físico cumple para la actividad turística. También hay que contemplar dicho medio como un capital ambiental, y el uso sostenible del medio será aquel que no altere ese capital ambiental. Los criterios que deben seguirse son:

- $\quad$ Actuar según el principio de precaución, tomando medidas respecto a la acción humana.

- Utilizar los recursos de manera que no transformen la calidad ambiental o, bien, hacerlo dentro de unos límites razonables.

- $\quad$ Corregir los daños causados mediante el principio de "quien contamina paga".

Por último, encontramos la sostenibilidad sociocultural que garantiza la compatibilidad entre el desarrollo turístico y la cultura y los valores de la población local, y que favorece la identidad de la comunidad autóctona (Puertas Cañaveral, 2007). En este sentido, se debe respetar la autenticidad sociocultural de las comunidades anfitrionas, conservar sus activos culturales, arquitectónicos y sus valores tradicionales, además de contribuir al entendimiento y la tolerancia intercultural (Canoves et al., 2006). Los criterios para hablar de una sostenibilidad social pueden ser:

- $\quad$ Que la actividad humana no conduzca a la aparición de desequilibrios o unafragmentación de la sociedad. 

turísticos.

Que la sociedad local se involucre en el desarrollo de los proyectos - $\quad$ Que los usuarios perciban la experiencia turística como satisfactoria.

Por otro lado, todas las medidas que se deben ejecutar en el turismo sostenible deben ser siempre a largo plazo. Así, según McIntyre(1993), durante la Conferencia Globe 90 , se desarrollaron un conjunto de principios que deben ser obligatoriamente tenidos en cuenta a la hora de comenzar un proyecto que pretenda ser sostenible (Puertas Cañaveral, 2007):

- Todos los stakeholders implicados deben seguir unos principios éticos querespeten la cultura y el medio ambiente del área, la economía y el modotradicional de vida.

- La planificación del turismo debe implicar la participación de todos losstakeholders.

- $\quad$ Debe aparecer una planificación del turismo desde la perspectiva del desarrollo sostenible.

- Debe existir una distribución equitativa de los beneficios entre los promotores y la poblaciónlocal.

- La información, investigación y comunicación de la naturaleza del turismoresulta prioritaria.

- La comunidad local debe implicarse en la planificación y desarrollo de losplanes locales, junto con los demás stakeholders.

- Todo proyecto debe conllevar un análisis integrado del medio ambiente,sociedad y economía.

- $\quad$ Los planes de desarrollo del turismo deben permitir a la comunidad local que sebeneficien de ellos.

Por tanto, el desarrollo sostenible en la actividad turística busca satisfacer las necesidades de los stakeholders presentes localizados en el destino, conservando los recursos y el medio natural para el disfrute de las generaciones futuras. En este mismo sentido, el turismo comunitario se configura como una herramienta adecuada para conseguir un desarrollo turístico sostenible en el destino.

\section{Conclusiones}

El turismo es una de las principales industrias económicas a nivel internacional. En este sentido, existen dos formas de turismo: El turismo de masas y los turismos alternativos a estos (Castillejo Canalejo et al., 2011). Este último grupo se caracteriza por desarrollarse de forma más sostenible. 
Así, el desarrollo sostenible busca satisfacer las necesidades de los presentes sin comprometer las de las generaciones futuras. Aplicada al turismo hace referencia a aquellas formas de turismo que satisfacen las necesidades de los residentes y los turistas, sin comprometer las necesidades de las generaciones futuras que habiten o visiten el destino turístico.

En este sentido, dentro de los pilares del desarrollo sostenible, encontramos el desarrollo socioeconómico y la conservación y protección hacia el medio ambiente. Estos pilares pueden aplicarse al turismo, y como tal, aparecen nuevas tipologías y formas de turismo que pueden desarrollarse de forma sostenible. Tal es el caso del turismo comunitario.

El turismo comunitario hace referencia a aquel tipo de turismo que se desarrolla en un destino específico, y donde los stakeholders locales son los encargados de planificar la actividad turística, siendo la comunidad local el principal eje de la actividad, y por consiguiente, el principal beneficiario. Además esta forma de turismo ayuda a conservar y poner en valor recursos de diversa índole: Patrimoniales, culturales o naturales.

Así, el turismo comunitario se plantea como una forma de turismo sostenible, que busca por un lado mejorar el desarrollo socioeconómico de la población localizada en las comunidades locales del destino, y por otro, pretende conservar y fomentar el respeto hacia los recursos naturales, patrimoniales y culturales, a través de su puesta en valor y de la implementación de nuevas formas sostenibles de gestión.

Esta forma de turismo adquiere más importancia en destinos subdesarrollados, donde temas relacionados con la generación de riqueza y conservación de los recursos se hacen vitales para la supervivencia de las comunidades locales, tanto del presente como también del futuro.

Así, el desarrollo del turismo comunitario en zonas desfavorecidas puede ayudar a generar recursos económicos en las poblaciones locales, contribuyendo así al desarrollo social y económico de la población y del destino. Además, esta forma de turismo puede generar una mayor concienciación de la comunidad local, fomentando aspectos como la conservación y respecto hacia la naturaleza y demás recursos existentes en el destino (cultura, patrimonio, etc.).

Un ejemplo de un país donde se podría implementar esta forma de turismo es República Dominicana, donde el turismo es una de las principales industrias (turismo de sol y playa), aunque, las poblaciones locales no se ven beneficiadas, debido a que mayoritariamente, las grandes empresas turísticas localizadas en el país son extranjeras. En este sentido, hay estudios que evidencia el gran potencial del país para desarrollar nuevas formas de turismo (Castellanos Verdugo y Orgaz Agüera, 2013), que además de ser más sostenibles que el turismo de masas, contribuirían al desarrollo de las comunidades locales y a la conservación de los recursos naturales. Y sin duda, una de estas formas de turismo con potencial para implementarse en el país es el turismo comunitario.

En este sentido, es necesario que el turismo comunitario se realice cumpliendo una serie de acciones, lo que fomentará el desarrollo sostenible en el destino. Así, y en primer lugar, se debe involucrar a la población local en el proceso de 
planificación y gestión de la actividad turística. En segundo lugar, es necesario elaborar un plan de desarrollo turístico del destino, que ayude a planificar de forma adecuada la actividad turística. En tercer lugar, se deben poner en valor aspectos como la cultura (gastronomía, bailes locales, folklore, etc.), el patrimonio histórico (edificios, industrias, etc.) y los recursos naturales (ríos, bosques, flora, fauna, etc.), y en todo momento, deben ser gestionados por las comunidades locales (o por lo menos, este grupo debe tener un gran peso en el reparto de beneficios por el desarrollo del turismo). Por último, y en cuarto lugar, es necesario un plan de concienciación y educación de la población local previo al inicio de la actividad turística, lo cual ayudará a formar mejores recurso humanos para el desempeño de la actividad turística en el destino. Estos planes educativos deben tener varios ejes, entre ellos cultura, medio ambiente, turismo, creación de empresas, calidad de servicio e idiomas, entre otros.

En definitiva, y a modo de conclusión final, el turismo comunitario, desarrollado desde una planificación previa donde la comunidad local sea uno de los ejes principales en la gestión y planificación del turismo, puede contribuir a desarrollar de forma sostenible el destino turístico, y sobre todo, esto tiene más peso en países subdesarrollados, donde esta forma de turismo puede ayudar a aliviar la pobreza y a conservar mejor los recursos (naturales, culturales y patrimoniales) del destino para el disfrute de las generaciones futuras, ya sean residentes o turistas.

\section{Referencias Bibliográficas}

a) (2011) ALAEDDINOGLU, F. y CAN, A. S. "Identification and classification of nature-based tourism resources: Western Lake Van basin, Turkey". Procedia: Social and Behavioral Sciences, vol. 19, pp.198-207.

b) (2008) BARAL, N.; STERN, M. J. y BHATTARAI, R. Contingent valuation of ecotourism in Annapurna conservation area, Nepal: Implications for sustainable park finance and local development. Ecological Economics, vol. 66, no 2-3, pp.218-227.

c) (2005) BLACKSTOCK, K. "A critical look at community based tourism". Community DevelopmentJournal, vol. 40, n 1, pp. 39-49.

d) (2004) BRINGAS RÁBAGO, N. e ISRAEL, J. "El turismo alternativo: una opción para el desarrollo local en dos comunidades indígenas de baja California". Economía, Sociedad y Territorio, vol. 4, nº 15, pp. 551-589.

e) (1987) BRUNDTLAND, C. G. Our Common Future. Oxford: The World Commission on Environmental Development.

f) (2006) CANOVES G.; VILLARINO, M. y HERRERA, L."Políticas públicas, turismo rural y sostenibilidad: difícil equilibrio". Boletín AGE, $n^{\circ} 41$, pp.199-217. 
g) (2012) CASAS JURADO, A. C.; SOLER DOMINGO, A. y JAIME PASTOR, V. "El turismo comunitario como instrumento de erradicación de la pobreza: Potencialidades para su desarrollo en Cuzco (Perú)". Cuadernos de Turismo, no 30 , pp. 91-108.

h) (2013) CASTELLANOS VERDUGO, M. y ORGAZ AGÜERA, F. "Potencialidades ecoturísticas de la República Dominicana". TURyDES: Revista de Investigación en Turismo y Desarrollo Local, vol. 6, n 14, pp. 1-9.

i) (2011) CASTILLEJO CANALEJO, A. M.; LÓPEZ-GUZMÁN, T. J. y MILLÁN VÁZQUEZ DE LA TORRE, M. G. Delimitación conceptual y consideraciones en torno al turismo industrial minero. TURyDES: Revista de Investigación en Turismo y Desarrollo Local, 4 (9): 1-15.

j) (2011) CHAKRAVARTY, S. y IRAZÁBAL, C. "Golden geese or white elephants? The paradoxes of world heritage sites and community-based tourism development in Agra, India". Community Development, vol. 42, nº 3, pp. 359-376.

k) (2007) CIOCE SAMPAIO, C. A.; BONA CARVALHO, M. y RIBEIRO DE ALMEIDA, F. H. "Community tourism: montnhabeija-flordourado pilot project (microbasin of the sagrado river, Morretes, Paraná)". Turismo-Visao e Açao, vol. $9, \mathrm{n}^{\circ} 2$, pp. 249-266.

I) (2012) CRUZ BLASCO, M. "Turismo, identidad y reivindicación sociocultural en Chile". Turismo y Sostenibilidad: $V$ Jornadas de Investigación en Turismo, Universidad de Sevilla, Sevilla, pp. 127-147.

m) (2003) DYER, P.; ABERDEEN, L. y SCHULER, S. "Tourism impacts on an Australian indigenous community: A Djabugay case study". Tourism Management, vol. 24, pp. 83-95.

n) (2011) FARRELLY, T. A. "Indigenous and democratic decision-making: issues from community-based ecotourism in the Boumā National Heritage Park, Fiji". Journal of Sustainable Tourism, vol. 19, n 7, pp. 817-835.

o) (2012) GIAMPICCOLI, A. y KALIS, J. H. "Tourism, Food, and Culture: Community-Based Tourism, Local Food, and Community Development in Mpondoland". Culture, Agriculture, Food and Environment, vol. 34, n 2, pp.101123.

p) (1998) GODFREY, K. B. Attitudes towards 'sustainable tourism' in the UK: a view from local government. Tourism Management, 19 (3): 213-224.

q) (2007) GUERREIRO MARCON, E. M. "O turismo como agente de desenvolvimento social e a comunidadeGuaraninasRuínas Jesuíticas de Sao MigueldasMissoes". PASOS:Revista de Turismo y Patrimonio Cultural, vol. 5, no 3, pp. 343-352.

r) (2012) HARUN, H.; HASSAN, R.; RAZZAQ, A.: RASID, A. y MUSTAFA, M. Z. "Building local capacities towards sustaining community based tourism development (CBET): experience from Miso Walal Homestay, Kinabantangan Sabah, Malaysia". Regional Conference on Higher Education-CommunityIndustry Engagement, 7-9 May 2012, Kuala Lumpur. 
s) (2006) HIWASAKI, L. "Community-based tourism: A pathway to sustainability for Japan's protected areas". Society and Natural Resources, vol. $19, \mathrm{n}^{\circ} 8$, pp. $675-692$.

t) (1997) HUNTER, C. "Sustainable tourism as an adaptive paradigm". Annals of Tourism Research, vol. 24, $\mathrm{n}^{\circ} 4$, pp. 850-867.

u) (2013) IORIO, M. y CORSALE, A. "Rural tourism and livelihood strategies in Romania". Journal of Rural Studies, vol. 26, n² 2, pp.152-162.

v) (2012) IORIO, M. Y WALL, G. "Behind the masks: Tourism and community in Sardinia". Tourism Management, vol. 33, nº 6, pp. 1440-1449.

w) (2012) ISHII, K. "The impact of ethnic tourism on hill tribes in Thailand". Annals of Tourism Research, vol. 39, $\mathrm{n}^{\circ}$ 1, pp. 290-310.

x) (2012) JALANI, J. O. "Local People's Perception on the Impacts and Importance of Ecotourism in Sabang, Palawan, Philippines". Procedia: Social and BehavioralSciences, vol. 57, pp. 247-254.

y) (2010) JARVIS, N.;WEEDEN, C. y SIMCOCK, N. "The Benefits and Challenges of Sustainable Tourism Certification: A Case Study of the Green Tourism Business Scheme in the West of England". Journal of Hospitality and Tourism Management,vol. 17, no 1, pp. 83-93.

z) (2008) SÁNCHEZ, J. P. Y RAMÍREZ VALVERDE, B. (2008): "El turismo rural como complemento al desarrollo territorial rural en zonas indígenas de México". Scripta Nova: Revista Electrónica de Geográfica y Ciencias Sociales, vol. 11, n² 236, pp. 1-15.

aa) (2010) LAPEYRE, R. "Community-based tourism as a sustainable solution to maximise impacts locally? The Tsiseb Conservancy case, Namibia". Development Southern Africa, vol. 27, $\mathrm{n}^{\circ} 5$.

bb) (2007) LEPP, A. "Residents attitudes towards tourism in Bigodi village, Uganda". Tourism Management, vol. 28, pp. 876-885.

cc) (2011) LÓPEZ-GUZMÁN, T.; BORGE, O. y CEREZO, J. M “Communitybased tourism and local socio-economic development: A case study in Cape Verde". African Journal of Business Management, vol. 5, nº 5, pp. 1608-1617.

dd) (2009a) LÓPEZ-GUZMÁN, T. y SÁNCHEZ CAÑIZARES, S. M. "Turismo comunitario y generación de riqueza en países en vías de desarrollo. Un estudio de caso en el Salvador". REVESCO: Revista de Estudios Cooperativos, $\mathrm{n}^{\circ} 30$, pp. 85-103.

ee) (2009b) LÓPEZ-GUZMÁN, T. Y SÁNCHEZ CAÑIZARES, S. M. "Desarrollo socioeconómico de las zonas rurales con base en el turismo comunitario. Un estudio de caso de Nicaragua". Cuadernos de Desarrollo Rural, n 6, pp. 81-97.

ff) (2012) MAK, A. H. N.; LUMBERS, M. y EVES, A. Globalisationand food consumption in tourism. Annals of Tourism Research, vol. 39, n 1, pp.171-196.

gg) (2006) MANYARA, G.; JONES, E. y BOTTERILL, D. "Tourism and poverty alleviation: The case for indigenous enterprise development in Kenya". Tourism, Culture and Communication, vol. 7, nº 1, pp. 19-37. 
hh) (2007) MANYARA, G. y JONES, E. "Community-based tourism enterprises development in Kenya: An exploration of their potential as avenues of poverty reduction". Journal of Sustainable Tourism, vol. $15, \mathrm{n}^{\circ} 6$, pp. 628644.

ii) (2005) MBAIWA, J. E. "Community-based tourism and the marginalized communities in Botswana: The case of the Basarna in Okavango Delta". En: Ryan, C. y Aicken, M., Indigenous tourism: The commodification and management of culture (pp: 87-109), Londres: Elsevier.

jj) (1993) MCINTYRE, G. Sustainable tourism development: Guide for local planners. Madrid: Organización Mundial delTurismo.

kk) (1985) MURPHY, P. E. Tourism: A community approach. Londres: Methuen.

II) (2009) NAE-WEN, K. y PEI-HUN, C. Quantifying energy use, carbon dioxide emission, and other environmental loads from island tourism based on a life cycle assessment approach. Journal of Cleaner Production, vol. 17, $\mathrm{n}^{\circ} 15$, pp.1324-1330.

mm) (2010) NELSON, F.; FOLEY, C.; FOLEY, L. S.; LEPOSO, A.; LOURE, E.; PETERSON, D.; PETERSON, M.; PETERSON, T.; SACHEDINA, H. y WILLIAMS, A. "Payments for Ecosystem Services as a Framework for Community-Based Conservation in Northern Tanzania". ConservationBiology, vol.24, $n^{\circ} 1$, pp. 78-85.

nn) (2002) OMT Tourism and Poverty Alleviation. Madrid: Organización Mundial del Turismo.

oo) (1998) PALOMO PÉREZ, S. "La aportación del turismo al desarrollo económico de los países en vías de desarrollo. El caso del mercado receptor de Bolivia". EstudiosTurísticos, nº 189, pp. 45-81.

pp) (2004) PATTERSON, T.; GULDEN, T.; COUSINS, K. y KRAEV, E. "Integrating environmental, social and economic systems: a dynamic model of tourism in Dominica". EcologicalModelling,vol. 175, n² 2, pp. 121-136.

qq) (2007) PUERTAS CAÑAVERAL, I. Ecoturismo en las reservas de la biosfera. Granada: Universidad de Granada.

rr) (2013) REIMER, J. K. y WALTER, P. "How do you know it when you see it? Community-based ecotourism in the Cardamom Mountains of southwestern Cambodia". Tourism Management, vol. 34, pp. 122-132.

sS) (2011) RUIZ BALLESTEROS, E. y CANTERO MARTÍN, P. A. "Entre Darwin, la baronesa y el cucuve. El desarrollo del turismo de base local en Floreana (Galápagos)". PASOS: Revista de Turismo y Patrimonio Cultural. Colección PASOS Edita, n 5, pp. 63-77

tt) (2008) RUIZ, E.; HERNÁNDEZ, M.; COCA, A.; CANTERO, P. y DEL CAMPO, A. "Turismo comunitario en Ecuador. Comprendiendo el communitybased tourism desde la comunidad". PASOS: Revista de Turismo y Patrimonio Cultural, vol. 6, n 3, pp. 399-418.

uu) (1998) SANCHO, A.Introducción al turismo.Madrid: Organización Mundial del Turismo. 
vv) (1999) SCHEYVENS, R. "Ecotourism and the Empowerment of Local Communities". Tourism Management, vol. 20, pp. 245-49.

ww) (2008) SHEN, F.; HUGHEY, K. F. D. y SIMMONS, D. G. Connecting the Sustainable Livelihoods Approach and Tourism: A Review of the Literature. Journal of Hospitality and Tourism Management, vol. 15, n 1, pp. 19-31.

xx) (2010) SOMMERVILLE, M.; JONES, J. P.; RAHAJAHARISON, M. y MILNER-GULLAND, E. J. "The role of fairness and benefit distribution in community-based Payment for Environmental Services interventions: A case study from Menabe, Madagascar". Ecological Economics, vol. 69, nº 6, pp. 1262-1271.

yy) (2009) STEWART, E. J. y DRAPER, D. "Reporting back research findings: a case study of community-based tourism research in northern Canada". Journal of Ecotourism, 8 (2): 128-143.

zz) (1999) TIMOTHY, D. J. y WHITE, K. "Community-based ecotourism development ion the periphery of Belize". Current Issues in Tourism, vol. 2, $\mathrm{n}^{\mathrm{o}}$ 2-3, pp. 226-243.

aaa) (2001) TOSUN, C. "Challenges of sustainable tourism development in the developing world: the case of Turkey". Tourism Management, vol. 22, $\mathrm{n}^{\circ} 3$, pp. 289-303.

bbb) (2009) TREJOS, B. "Redes de apoyo comunitario al turismo comunitario en Costa Rica". TURyDES: Revista de Investigación en Turismo y Desarrollo Local, vol. 2, nº 6 , pp. 1-15.

ccc) (2010) TREJOS, B. y MATARRITA-CASCANTE, D. "Theoretical Approximations to Community-Based Tourism: Case Studies from Costa Rica", Review of Tourism Research, vol. 8, nº 6, pp. 157-178.

ddd) (2013) WALIGO, V. M.; CLARKE, J. y HAWKINS, R. "Implementing sustainable tourism: A multi-stakeholder involvement management framework". Tourism Management, vol. 36, pp.342-353.

eee) (2001) WORLD WIDE FUND FOR NATURE Directrices para el desarrollo del turismo comunitario. United Kingdom: WWF.

fff) (1998) WYLLIE, R. W. (1998): "Hana revisited: development and controversy in a Hawaiian tourism community". Tourism Management, vol. 19, $\mathrm{n}^{\circ} 2$, pp. 171-178.

ggg) (2007) YING, T. y ZHOU, Y. (2007): "Community, governments and external capitals in China's rural cultural tourism: A comparative study of two adjacent villages". Tourism Management, vol. 28, n 1, pp. 96-107.

hhh) (2007) ZORN, E. y FARTHING, L. C. "Communitarian tourism. Hosts and mediators in Peru". Annals of Tourism Research, vol. 34, nº 3, pp. 673-689. 